\title{
On Regular Co-Medial Algebras
}

\author{
Amir Ehsani (Corresponding author) \\ Department of Mathematics, Mahshahr Branch, Islamic Azad University \\ Mahshahr 63519, Iran
}

Tel: 98-0936-435-5573 E-mail: a.ehsani@mahshahriau.ac.ir

Received: January 11, 2012 Accepted: January 29, 2012 Published: April 1, 2012

doi:10.5539/jmr.v4n2p101 URL: http://dx.doi.org/10.5539/jmr.v4n2p101

\begin{abstract}
In this paper we define the concepts of co-medial algebra and regular algebra and we will show that for a regular co-medial algebra $(A, f, g)$, there exist a commutative semigroup $(A,+)$, such that the operations $f, g$ have the linear representation on $(A,+)$. As a consequence of above result, we have the linear representation of an $n$-ary medial groupoid with an regular element, which was obtained by (Evans, T., 1963, p. 331-349).
\end{abstract}

Keywords: Co-medial algebra, Regular algebra, Medial algebra, Regular element

\section{Introduction}

The medial property was studied initially by (Kolmogorov, A., 1930; Nagumo, M., 1930; Bruck, R. H., 1944; Aczél, J., 1947; Hosszu, M., 1953). An algebra $A=(A, F)$, (without nollary operations) is called medial (entropic, abelian) if it satisfies the identity of mediality:

$$
g\left(f\left(x_{11}, \ldots, x_{n 1}\right), \ldots, f\left(x_{1 m}, \ldots, x_{n m}\right)\right)=f\left(g\left(x_{11}, \ldots, x_{1 m}\right), \ldots, g\left(x_{n 1}, \ldots, x_{n m}\right)\right),
$$

for every n-ary $f \in F$ and m-ary $g \in F$ (Kurosh, A. G., 1947). The n-ary operation, $f$, is called idempotent if $f(x, \ldots, x)=$ $x$, for every $x \in A$. The algebra $A=(A, F)$ is called idempotent, if every operation $f \in F$ is idempotent. An idempotent medial algebra is a mode (Romanowska, A. \& Smith, J. D. H., 2002). In other words, the algebra, $A$, is medial if it satisfies the hyperidentity of mediality (Movsisyan, Yu. M., 1986; Movsisyan, Yu. M., 1990; Movsisyan, Yu. M., 1998). Note that a groupoid is medial iff it satisfies the identity of mediality (Ježek, J., Kepka, T., 1983): xy.uv $\approx x u . y v$.

Let $g$ and $f$ be m-ary and n-ary operations on the set, $A$. We say that the pair of operations, $(f, g)$, is medial (entropic), if the identity (1) holds in the algebra $A=(A, f, g)$. Characterization of medial pair of binary quasigroup operations, obtained in (Movsisyan, Yu. M., 1999) (Also see Movsisyan, Yu. M. \& Nazari, E., 2011).

The n-ary groupoid $(A, f)$, is medial if it is satisfies the following identity:

$$
f\left(f\left(x_{11}, \ldots, x_{n 1}\right), \ldots, f\left(x_{1 n}, \ldots, x_{n n}\right)\right)=f\left(f\left(x_{11}, \ldots, x_{1 n}\right), \ldots, f\left(x_{n 1}, \ldots, x_{n n}\right)\right) .
$$

Let $A=(A, F)$ be an algebra and $f \in F$. We say that the element $e$, is the unit element for the operation $f$, if:

$$
f(x, e, \ldots, e)=f(e, x, e, \ldots, e)=\cdots=f(e, \ldots, e, x)=x,
$$

for every $x \in A$. The element $e$ is an unit element for the algebra $(A, F)$, if it is an unit for every operation $f \in F$.

The element $e$, is an idempotent element for the operation $f \in F$, if: $f(e, \ldots, e)=e$. An element $e$ is idempotent element for the algebra $(A, F)$, if it is an idempotent element for every operation $f \in F$.

Definition 1.1 Let $g$ and $f$ are n-ary operations on the set $A$. We say that the pair of n-ary operations $(f, g)$, is co-medial pair operation if the following identity holds in the algebra $A=(A, f, g)$ :

$$
g\left(f\left(x_{11}, \ldots, x_{n 1}\right), \ldots, f\left(x_{1 n}, \ldots, x_{n n}\right)\right)=g\left(f\left(x_{11}, \ldots, x_{1 n}\right), \ldots, f\left(x_{n 1}, \ldots, x_{n n}\right)\right) .
$$

The algebra $A=(A, F)$ is called co-medial algebra if, every pair of operations $f, g \in F$ with the same arity is a co-medial pair operation. If $f=g$ then, the co-medial pair operation $(f, f)$ is a medial pair operation.

There exist various algebraic characterizations of different classes of n-ary operations (see for example Dudek, W. A. \& Trokhimenko, V. S., 2010). In this article we investigate a generalizatin of algebras with a medial operation, which we called a co-medial algebras. 
Definition 1.2 Let $(f, g)$ be a pair of n-ary operations of the algebra, $(A, F)$. For any element, $e$, of $A$, let $\alpha_{1}, \ldots, \alpha_{n}$ and $\beta_{1}, \ldots, \beta_{n}$ be mappings of $A$ into $A$ defined by

$$
\begin{aligned}
\alpha_{i}: x \mapsto f(e, \ldots, e, x, e, \ldots, e), \\
\beta_{i}: x \mapsto g(e, \ldots, e, x, e, \ldots, e),
\end{aligned}
$$

with $x$ at the $i$-th place. We call $\alpha_{i}$ the $i$-th translation by e with respect to $f$. An element $e$ is called $i-r e g u l a r ~ e l e m e n t$ with respect to $f$ if $\alpha_{i}$ is a bijection. The similar definitions go with $g$. An element $e$, is called $i-$ regular for the pair operation $(f, g)$ if, it is $i$-regular element with respect to the both operations $f$ and $g$. The element $e$ is called $i-$ regular for the algebra $(A, F)$, if it is $i$-regular for every pair operations $f, g \in F$.

\section{Preliminary Results}

Definition 2.1 Let $f$ be an $n$-ary operation and $J$ be a non-empty subset of $\{1,2, \ldots, n\}$, we will say that the element $e$ is $J$-regular with respect to the operation $f$, if $e$ is a $j$-regular element with respect to $f$, for all $j \in J$. The element $e$ is $J$-regular element for the algebra $(A, F)$, if $e$ is a $J$-regular element with respect to every $f \in F$.

Definition 2.2 Let $f, g \in F$ be n-ary operations $(2 \leq n), \emptyset \neq J \subseteq\{1,2, \ldots, n\}$ and $a_{1}, \ldots, a_{i-1}, a_{i+1}, \ldots, a_{n}$ are $J$-regular elements of the algebra $(A, f, g)$ (where $J$ contains at leas two elements). The pair operation $(f, g)$ is $(i, J)$-regular pair operation (where $i \in J$ ), if for every $x \in A$ we have the following equality:

$$
f\left(a_{1}, \ldots, a_{i-1}, x, a_{i+1}, \ldots, a_{n}\right)=g\left(a_{1}, \ldots, a_{i-1}, x, a_{i+1}, \ldots, a_{n}\right)
$$

The pair operation $(f, g)$ is a $J$-regular if $(f, g)$ is $(i, J)$-regular for every $i \in J$. The pair operation $(f, g)$ is regular if $(f, g)$ is a $J$-regular pair operation for some $\emptyset \neq J \subseteq\{1,2, \ldots, n\}$ (where $J$ contains at leas two elements). An algebra $(A, F)$ is called regular if every pair operation of $(A, F)$ be a regular pair operation. The equality (3) is a co-identity in the sence of (Movsisyan, Yu. M., 1986).

Lemma 2.3 Let $(A, f, g)$ be a regular co-medial algebra with an idempotent $i$-regular element, then $f=g$.

Proof: Let $(A, f, g)$ be a regular co-medial algebra with an idempotent $i$-regular element $e$, then for every $x_{1}, \ldots, x_{n} \in A$ we have:

$$
\begin{aligned}
& \beta_{i} g\left(x_{1}, \ldots, x_{n}\right) \\
& =g\left(e, \ldots, e, g\left(x_{1}, \ldots, x_{n}\right), e, \ldots, e\right) \\
& =g\left(g(e, \ldots, e), \ldots, g\left(x_{1}, \ldots, x_{n}\right), \ldots, g(e, \ldots, e)\right) \\
& =g\left(g\left(e, \ldots, e, x_{1}, e, \ldots, e\right), \ldots, g\left(e, \ldots, e, x_{i}, e, \ldots, e\right), \ldots, g\left(e, \ldots, e, x_{n}, e, \ldots, e\right)\right) \\
& =g\left(\beta_{i} x_{1}, \ldots, \beta_{i} x_{n}\right) .
\end{aligned}
$$

So, $\beta_{i}^{-1} g\left(x_{1}, \ldots, x_{n}\right)=g\left(\beta_{i}^{-1} x_{1}, \ldots, \beta_{i}^{-1} x_{n}\right)$. Also, since $(A, f, g)$ is a regular algebra with the $i-$ regular element $e, \alpha_{i}=\beta_{i}$. Therefor, $\alpha_{i}^{-1}=\beta_{i}^{-1}$ and we have:

$$
\begin{aligned}
& g\left(x_{1}, \ldots, x_{n}\right) \\
& =f\left(e, \ldots, e, \alpha_{i}^{-1} g\left(x_{1}, \ldots, x_{n}\right), e, \ldots, e\right) \\
& =f\left(e, \ldots, e, \beta_{i}^{-1} g\left(x_{1}, \ldots, x_{n}\right), e, \ldots, e\right) \\
& =f\left(g(e, \ldots, e), \ldots, g\left(\beta_{i}^{-1} x_{1}, \ldots, \beta_{i}^{-1} x_{n}\right), \ldots, g(e, \ldots, e)\right) \\
& =f\left(g\left(e, \ldots, e, \beta_{i}^{-1} x_{1}, e, \ldots, e\right), \ldots, g\left(e, \ldots, e, \beta_{i}^{-1} x_{n}, e, \ldots, e\right)\right) \\
& =f\left(x_{1}, \ldots, x_{n}\right) .
\end{aligned}
$$

Hence, $f=g$.

The following characterization of a medial n-ary groupoid with an idempotent $J$-regular element, obtained by (Evans, T., 1963).

Theorem 2.4 Let $(A, f)$ be an $n$-ary medial groupoid with a J-regular idempotent element e (where $J \subseteq\{1,2, \ldots, n\}$ contains at leas two elements), then there exists a commutative semigroup $(A,+)$ with the unit element $e$, such that the operation $f$ has the following linear representation

$$
f\left(x_{1}, \ldots, x_{n}\right)=\alpha_{1} x_{1}+\cdots+\alpha_{n} x_{n},
$$

where $\alpha_{1}, \ldots, \alpha_{n}$, are pairwise commuting endomorphisms of $(A,+), n \geq 2$. If $i, j \in J$, then $\alpha_{i}, \alpha_{j}$ are automorphisms of $(A,+)$. 
Proof: See, Evans, T., 1963.

Corollary 2.5 Let $(A, f, g)$ be a regular co-medial $n$-ary algebra with an idempotent J-regular element e (where $J \subseteq$ $\{1,2, \ldots, n\}$ contains at leas two elements), then there exists a commutative semigroup $(A,+)$ with the unit element $e$, such that

$$
g\left(x_{1}, \ldots, x_{n}\right)=f\left(x_{1}, \ldots, x_{n}\right)=\alpha_{1} x_{1}+\cdots+\alpha_{n} x_{n},
$$

where $\alpha_{1}, \ldots, \alpha_{n}$, are pairwise commuting endomorphisms of $(A,+), n \geq 2$. If $i, j \in J$, then $\alpha_{i}, \alpha_{j}$ are automorphisms of $(A,+)$.

Proof: By Lemma 2.3, $f=g$. Therefor, the algebra $(A, f, g)$ is an $n$-ary medial groupoid, so we can use the Theorem 2.4 .

We have described in the Corollary 2.5 structure of the regular co-medial algebra $(A, f, g)$ containing an idempotent $J$-regular element. The purpose of this section is to obtain sufficient properties of finite co-medial algebras to enable us to weaken considerably, in this finite case, the assumptions we need for characterizing regular co-medial algebras which do not contain an idempotent element.

We use continually the following lemma, the proof of which we omit.

Lemma 2.6 Let $(A, F)$ be a finite algebra and $a_{1}, \ldots, a_{i-1}, a_{i+1}, \ldots, a_{n}$ are elements of $A$ and $f \in F$, if for all $x, y \in A$,

$$
f\left(a_{1}, \ldots, a_{i-1}, x, a_{i+1}, \ldots, a_{n}\right)=f\left(a_{1}, \ldots, a_{i-1}, y, a_{i+1}, \ldots, a_{n}\right),
$$

implies that $x=y$, then for any $b \in A$ there is a unique element $x \in A$ such that

$$
f\left(a_{1}, \ldots, a_{i-1}, x, a_{i+1}, \ldots, a_{n}\right)=b .
$$

Lemma 2.7 Let $(A, f, g)$ be a finite co-medial algebra with an $J$-regular element e. If $f\left(a_{1}, \ldots, a_{n}\right)=e$ and $g\left(c_{1}, \ldots, c_{n}\right)=$ $e$ then, for each $i, a_{i}$, is a J-regular element with respect to the operation $f$ and $c_{i}$ is a J-regular element with respect to the operation $g$.

Proof: Let $x, y \in A$ and $i \in J$, such that

$$
f\left(a_{i}, \ldots, a_{i}, x, a_{i}, \ldots, c_{i}\right)=f\left(a_{i}, \ldots, a_{i}, y, a_{i}, \ldots, a_{i}\right)
$$

then, we have:

$$
\begin{gathered}
g(f\left(a_{1}, \ldots, e, \ldots, a_{1}\right), \ldots, \overbrace{f(a_{i}, \ldots, \underbrace{x}_{\underbrace{x}_{i-t h}}, \ldots, a_{i})}^{i_{\text {th }}^{i-t h}}, \ldots, f\left(a_{n}, \ldots, e, \ldots, a_{n}\right))= \\
g(f\left(a_{1}, \ldots, e, \ldots, a_{1}\right), \ldots, \overbrace{f(a_{i}, \ldots, \underbrace{y}_{i-t h}, \ldots, a_{i})}^{\underbrace{\text { th }}}, \ldots, f\left(a_{n}, \ldots, e, \ldots, a_{n}\right)) .
\end{gathered}
$$

So, by co-mediality we have:

$$
\begin{aligned}
& g(f\left(a_{1}, \ldots, a_{n}\right), \ldots, \overbrace{f(e, \ldots, \underbrace{x}_{i-t h}, \ldots, e)}^{i-t h}, \ldots, f\left(a_{1}, \ldots, a_{n}\right))= \\
& g(f\left(a_{1}, \ldots, a_{n}\right), \ldots, \overbrace{f(e, \ldots, \underbrace{y}_{i-t h}, \ldots, e)}^{i-t h}, \ldots, f\left(a_{1}, \ldots, a_{n}\right)), \\
& g(e, \ldots, f(e, \ldots, x, \ldots, e), \ldots, e)=g(e, \ldots, f(e, \ldots, y, \ldots, e), \ldots, e) .
\end{aligned}
$$

Thus, by applying regularity of the element $e$, we have: $x=y$.

Similarly, if

$$
g\left(c_{i}, \ldots, c_{i}, x, c_{i}, \ldots, c_{i}\right)=g\left(c_{i}, \ldots, c_{i}, y, c_{i}, \ldots, c_{i}\right),
$$

then, $x=y$. By Lemma 2.6, this concludes the proof.

Lemma 2.8 Let $(A, f, g)$ be a finite co-medial algebra. If $e$ is an $i$-regular element in $(A, f, g)$, then so are $f(e, \ldots, e)$ and $g(e, \ldots, e)$. 
Proof: Let $x, y \in A$, such that

$$
g(f(e, \ldots, e), \ldots, x, \ldots, f(e, \ldots, e))=g(f(e, \ldots, e), \ldots, y, \ldots, f(e, \ldots, e)),
$$

and $t_{1}$ be the element of $A$ satisfying

$$
f\left(e, \ldots, t_{1}, \ldots, e\right)=e,
$$

with $t_{1}$ at the $i-t h$ place. By Lemma 2.7, $t_{1}$ is regular element with respect to $f$. So, if

$$
\begin{aligned}
& f\left(t_{1}, \ldots, x_{1}, \ldots, t_{1}\right)=x, \\
& f\left(t_{1}, \ldots, y_{1}, \ldots, t_{1}\right)=y,
\end{aligned}
$$

with $x_{1}, y_{1}$ at the $i-t h$ place, then we have:

$$
\begin{aligned}
& g(f(e, \ldots, e), \ldots, \overbrace{f\left(t_{1}, \ldots, x_{1}, \ldots, t_{1}\right)}^{i-t h}, \ldots, f(e, \ldots, e))= \\
& g\left(f(e, \ldots, e), \ldots, f\left(t_{1}, \ldots, y_{1}, \ldots, t_{1}\right), \ldots, f(e, \ldots, e)\right) .
\end{aligned}
$$

So, by co-mediality we have:

$$
\begin{aligned}
& g(f\left(e, \ldots, t_{1}, \ldots, e\right), \ldots, \overbrace{f\left(e, \ldots, x_{1}, \ldots, e\right)}^{i-t h}, \ldots, f\left(e, \ldots, t_{1}, \ldots, e\right))= \\
& g(f\left(e, \ldots, t_{1}, \ldots, e\right), \ldots, \overbrace{f\left(e, \ldots, y_{1}, \ldots, e\right)}^{i-t h}, \ldots, f\left(e, \ldots, t_{1}, \ldots, e\right)) .
\end{aligned}
$$

Since, $e$ is an $i$-regular element of $(A, f, g), x_{1}=y_{1}$. Hence, $x=y$. By Lemma 2.6, this concludes $f(e, \ldots, e)$ is an $i$-regular element with respect to the operation $g$. Similarly, $f(e, \ldots, e)$ is an $i$-regular element with respect to the operation $f$, and $g(e, \ldots, e)$ is an $i$-regular element with respect to the both operations $f, g$.

Lemma 2.9 Let $(A, f, g)$ be a finite co-medial algebra with $n$-ary operations, and $a_{1}, \ldots, a_{i-1}, a_{i+1}, \ldots, a_{n}$ are $J-r e g u l a r$ elements of the algebra $(A, f, g)$ (where $J \subset\{1,2, \ldots, n\}$ contains at leas two elements). Then, for every $b \in A$, there are unique $x_{1}, x_{2} \in A$ such that

$$
\begin{array}{r}
f\left(a_{1}, \ldots, a_{i-1}, x_{1}, a_{i+1}, \ldots, a_{n}\right)=b, \\
g\left(a_{1}, \ldots, a_{i-1}, x_{2}, a_{i+1}, \ldots, a_{n}\right)=b .
\end{array}
$$

Proof: Let $x_{1}, y_{1} \in A$, such that

$$
f\left(a_{1}, \ldots, a_{i-1}, x_{1}, a_{i+1}, \ldots, a_{n}\right)=f\left(a_{1}, \ldots, a_{i-1}, y_{1}, a_{i+1}, \ldots, a_{n}\right),
$$

we will prove that, $x_{1}=y_{1}$.

For $k=1, \ldots, i-1, i+1, \ldots, n$ let,

$$
f\left(a_{k}, \ldots, t_{k}, \ldots, a_{k}\right)=a_{1},
$$

with $t_{k}$ at the $j-t h$ place. Then, we have:

$$
\begin{gathered}
g(f\left(a_{1}, \ldots, a_{i-1}, a_{1}, a_{i+1}, \ldots, a_{n}\right), \ldots, \overbrace{f\left(a_{1}, \ldots, a_{i-1}, x_{1}, a_{i+1}, \ldots, a_{n}\right)}^{i-t h}, \ldots \\
\ldots, \overbrace{f\left(t_{1}, \ldots, t_{i-1}, a_{1}, t_{i+1}, \ldots, t_{n}\right)}^{j-t h}, \ldots, f\left(a_{1}, \ldots, a_{i-1}, a_{1}, a_{i+1}, \ldots, a_{n}\right))= \\
g(f\left(a_{1}, \ldots, a_{i-1}, a_{1}, a_{i+1}, \ldots, a_{n}\right), \ldots, \overbrace{f\left(a_{1}, \ldots, a_{i-1}, y_{1}, a_{i+1}, \ldots, a_{n}\right)}^{i-t h}, \ldots \\
\ldots, \overbrace{f\left(t_{1}, \ldots, t_{i-1}, a_{1}, t_{i+1}, \ldots, t_{n}\right)}^{j-t h}, \ldots, f\left(a_{1}, \ldots, a_{i-1}, a_{1}, a_{i+1}, \ldots, a_{n}\right)) .
\end{gathered}
$$

So, by co-mediality, we have:

$$
\begin{aligned}
& g(f\left(a_{1}, \ldots, t_{1}, \ldots, a_{1}\right), \ldots, \overbrace{f\left(a_{1}, \ldots, x_{1}, \ldots, a_{1}\right)}^{i-t h}, \ldots, f\left(a_{n}, \ldots, t_{n}, \ldots, a_{n}\right))= \\
& g(f\left(a_{1}, \ldots, t_{1}, \ldots, a_{1}\right), \ldots, \overbrace{f\left(a_{1}, \ldots, y_{1}, \ldots, a_{1}\right)}^{i-t h}, \ldots, f\left(a_{n}, \ldots, t_{n}, \ldots, a_{n}\right)), \\
& g(a_{1}, \ldots, \overbrace{f\left(a_{1}, \ldots, x_{1}, \ldots, a_{1}\right)}^{i-t h}, \ldots, a_{1})=g(a_{1}, \ldots, \overbrace{f\left(a_{1}, \ldots, y_{1}, \ldots, a_{1}\right)}^{i-t h}, \ldots, a_{1}),
\end{aligned}
$$


Two applications of the i-regularity of $a_{1}$ yield $x_{1}=y_{1}$. Similarly, if

$$
g\left(a_{1}, \ldots, a_{i-1}, x_{2}, a_{i+1}, \ldots, a_{n}\right)=g\left(a_{1}, \ldots, a_{i-1}, y_{2}, a_{i+1}, \ldots, a_{n}\right),
$$

then, $x_{2}=y_{2}$.

Specifically, from the above lemmas, we known if the finite co-medial algebra $(A, f, g)$ contains an element $e$ which is $J$-regular element, then

1. $f(e, \ldots, e)$ and $g(e, \ldots, e)$ are also $J$-regular element of $(A, f, g)$,

2. there are unique elements $t_{1}, t_{2} \in A$, which are regular with respect to $f$ and $g$ (respectively), such that (for $i<j$ )

$$
\begin{gathered}
f\left(e, \ldots, e, f(e, \ldots, e), e, \ldots, e, t_{1}, e, \ldots, e\right)=e \\
g\left(e, \ldots, e, g(e, \ldots, e), e, \ldots, e, t_{2}, e, \ldots, e\right)=e
\end{gathered}
$$

with $f(e, \ldots, e)$ and $g(e, \ldots, e)$ at the $i-t h$ places and $t_{1}, t_{2}$ at the $j-t h$ places.

3. for every $b \in A$, there are unique elements $x_{1}, x_{2} \in A$ such that

$$
\begin{gathered}
f\left(e, \ldots, e, x_{1}, e, \ldots, e, t_{1}, e, \ldots, e\right)=b \\
g\left(e, \ldots, e, x_{2}, e, \ldots, e, t_{2}, e, \ldots, e\right)=b
\end{gathered}
$$

with $x_{1}, x_{2}$ at the the $i-t h$ places and $t_{1}, t_{2}$ at the $j-t h$ places, where $t_{1}, t_{2}$ are the elements described in (2).

It is easy to prove that in the finite co-medial algebra $(A, f, g)$ the set of $J$-regular elements is closed under the operations $f, g$. Thus, if the finite co-medial algebra $(A, f, g)$, contains at least one $J$-regular element, then the algebra $(A, f, g)$, contains an $J$-regular subalgebra, where by $J$-regular subalgebra of a co-medial algebra, finite or infinite, we mean a subalgebra of $J$-regular elements such that if $a_{1}, \ldots, a_{i-1}, a_{i+1}, \ldots, a_{n}$ belong to the subalgebra, then there are the unique elements $x_{1}, x_{2} \in A$, for each $b \in A$ such that

$$
\begin{gathered}
f\left(a_{1}, \ldots, a_{i-1}, x_{1}, a_{i+1}, \ldots, a_{n}\right)=b, \\
g\left(a_{1}, \ldots, a_{i-1}, x_{2}, a_{i+1}, \ldots, a_{n}\right)=b .
\end{gathered}
$$

Furthermore, if $b$ is in the subalgebra, so are $x_{1}, x_{2}$.

\section{The Structure of Regular Co-Medial Algebras}

We discuss in this section the structure of a regular co-medial algebra $(A, f, g)$ which does not contain an idempotent element. We construct new operations $f^{*}, g^{*}$ on $A$ in terms of $f, g$, such that $\left(f^{*}, g^{*}\right)$ is a co-medial pair operation, and the co-medial algebra $\left(A, f^{*}, g^{*}\right)$, contains an idempotent element. If certain regularity conditions are assumed for $(A, f, g)$, then this idempotent element is also a $J$-regular element in $\left(A, f^{*}, g^{*}\right)$ and hence we are able to use the corollary 2.5 to describe the structure of the pair operation $\left(f^{*}, g^{*}\right)$.

Lemma 3.1 Let $(A, f, g)$ be a co-medial algebra and $\pi, \rho$ be permutations of $\{1,2, \ldots, n\}$, then the pair operation $\left(f^{*}, g^{*}\right)$ is co-medial, where

$$
\begin{array}{r}
f^{*}\left(x_{1}, x_{2}, \ldots, x_{n}\right)=f\left(x_{\pi 1}, x_{\pi 2}, \ldots, x_{\pi n}\right), \\
g^{*}\left(x_{1}, x_{2}, \ldots, x_{n}\right)=g\left(x_{\rho 1}, x_{\rho 2}, \ldots, x_{\rho n}\right) .
\end{array}
$$

Proof: For $x_{i j} \in A$, since $(A, f, g)$ is a co-medial algebra, we have:

$$
\begin{aligned}
& g^{*}\left(f^{*}\left(x_{11}, \ldots, x_{n 1}\right), \ldots, f^{*}\left(x_{1 n}, \ldots, x_{n n}\right)\right) \\
& =g\left(f\left(x_{\rho 1 \pi 1}, \ldots, x_{\rho n \pi 1}\right), \ldots, f\left(x_{\rho 1 \pi n}, \ldots, x_{\rho n \pi n}\right)\right) \\
& =g\left(f\left(x_{\rho 1 \pi 1}, \ldots, x_{\rho 1 \pi n}\right), \ldots, f\left(x_{\rho n \pi 1}, \ldots, x_{\rho n \pi n}\right)\right) \\
& =g^{*}\left(f^{*}\left(x_{11}, \ldots, x_{1 n}\right), \ldots, f\left(x_{n 1}, \ldots, x_{n n}\right)\right) .
\end{aligned}
$$

Lemma 3.2 Let $(A, f, g)$ be a finite co-medial algebra and $a_{1}, \ldots, a_{i-1}, a_{i+1}, \ldots, a_{n}$ are elements of $A$. Then, the pair operation defined by the following

$$
\begin{array}{r}
f^{*}\left(x_{1}, \ldots, x_{n}\right)=f\left(a_{1}, \ldots, a_{i-1}, f\left(x_{1}, \ldots, x_{n}\right), a_{i+1}, \ldots, a_{n}\right), \\
g^{*}\left(x_{1}, \ldots, x_{n}\right)=g\left(a_{1}, \ldots, a_{i-1}, g\left(x_{1}, \ldots, x_{n}\right), a_{i+1}, \ldots, a_{n}\right),
\end{array}
$$


is a co-medial pair operation on A.

Proof: In view of Lemma 3.1, it is sufficient to prove this for $i=1$.

$$
\begin{aligned}
& g^{*}\left(f^{*}\left(x_{11}, \ldots, x_{1 n}\right), \ldots, f^{*}\left(x_{n 1}, \ldots, x_{n n}\right)\right) \\
& =g\left(g\left(f^{*}\left(x_{11}, \ldots, x_{1 n}\right), \ldots, f^{*}\left(x_{n 1}, \ldots, x_{n n}\right)\right), a_{2}, \ldots, a_{n}\right) .
\end{aligned}
$$

But,

$$
\begin{aligned}
& g\left(f^{*}\left(x_{11}, \ldots, x_{1 n}\right), \ldots, f^{*}\left(x_{n 1}, \ldots, x_{n n}\right)\right) \\
& =g\left(f\left(f\left(x_{11}, \ldots, x_{1 n}\right), a_{2}, \ldots, a_{n}\right), \ldots, f\left(f\left(x_{n 1}, \ldots, x_{n n}\right), a_{2}, \ldots, a_{n}\right)\right) \\
& =g\left(f\left(f\left(x_{11}, \ldots, x_{n 1}\right), \ldots, f\left(x_{1 n}, \ldots, x_{n n}\right)\right), f\left(a_{2}, \ldots, a_{2}\right), \ldots, f\left(a_{n}, \ldots, a_{n}\right)\right) \\
& =g\left(f\left(f\left(x_{11}, \ldots, x_{n 1}\right), \ldots, f\left(x_{1 n}, \ldots, x_{n n}\right)\right), f\left(a_{2}, \ldots, a_{2}\right), \ldots, f\left(a_{n}, \ldots, a_{n}\right)\right) \\
& =g\left(f\left(f\left(x_{11}, \ldots, x_{n 1}\right), x_{2}, \ldots, a_{n}\right), \ldots, f\left(f\left(x_{1 n}, \ldots, x_{n n}\right), a_{2}, \ldots, a_{n}\right)\right) \\
& =g\left(f^{*}\left(x_{11}, \ldots, x_{n 1}\right), \ldots, f^{*}\left(x_{1 n}, \ldots, x_{n n}\right)\right),
\end{aligned}
$$

since, $(A, f, g)$ is co-medial.

So,

$$
\begin{aligned}
& g^{*}\left(f^{*}\left(x_{11}, \ldots, x_{1 n}\right), \ldots, g\left(x_{n 1}, \ldots, x_{n n}\right)\right) \\
& =g\left(g\left(f^{*}\left(x_{11}, \ldots, x_{n 1}\right), \ldots, f^{*}\left(x_{1 n}, \ldots, x_{n n}\right)\right), a_{2}, \ldots, a_{n}\right) \\
& =g^{*}\left(f^{*}\left(x_{11}, \ldots, x_{n 1}\right), \ldots, f^{*}\left(x_{1 n}, \ldots, x_{n n}\right)\right) .
\end{aligned}
$$

Hence, $\left(f^{*}, g^{*}\right)$ is a co-medial pair operation.

Lemma 3.3 Let $(A, f, g)$ be a co-medial algebra and, $e, t_{1}, t_{2} \in A$ such that

$$
\begin{gathered}
f\left(e, \ldots, e, f(e, \ldots, e), e, \ldots, e, t_{1}, e, \ldots, e\right)=e, \\
g\left(e, \ldots, e, g(e, \ldots, e), e, \ldots, e, t_{2}, e, \ldots, e\right)=e .
\end{gathered}
$$

Then, the pair operation $\left(f^{*}, g^{*}\right)$ on A, defined by the following

$$
\begin{gathered}
f^{*}\left(x_{1}, \ldots, x_{n}\right)=f\left(e, \ldots, e, f\left(x_{1}, \ldots, x_{n}\right), e, \ldots, e, t_{1}, e, \ldots, e\right), \\
g^{*}\left(x_{1}, \ldots, x_{n}\right)=g\left(e, \ldots, e, g\left(x_{1}, \ldots, x_{n}\right), e, \ldots, e, t_{2}, e, \ldots, e\right),
\end{gathered}
$$

with $f\left(x_{1}, \ldots, x_{n}\right)$ and $g\left(x_{1}, \ldots, x_{n}\right)$ at the $i-t h$ place and $t_{1}, t_{2}$ at the $j-$ th place, is a co-medial pair operation with e as an idempotent element.

Proof: This follows immediately by Lemma 3.2 and direct computation of $f^{*}(e, \ldots, e)$ and $g^{*}(e, \ldots, e)$.

Lemma 3.4 Let $(A, f, g)$ be regular co-medial algebra, then there is a commutative semigroup $(A,+)$, such that

$$
\begin{aligned}
& f\left(x_{1}, \ldots, x_{n}\right)=\varphi\left(\alpha_{1} x_{1}+\cdots+\alpha_{n} x_{n}\right), \\
& g\left(x_{1}, \ldots, x_{n}\right)=\psi\left(\alpha_{1} x_{1}+\cdots+\alpha_{n} x_{n}\right),
\end{aligned}
$$

where, $\alpha_{1}, \ldots, \alpha_{n}$, are pairwise commuting endomorphisms of $(A,+)$ and $\varphi, \psi$ are bijections on $A$, for $n \geq 2$.

Proof: If $J \subseteq\{1,2, \ldots, n\}, i, j \in J$ and $e$ is a $J$-regular element in $(A, f, g)$, then by results of the preceding section, there are $J$-regular elements $t_{1}, t_{2}$, such that

$$
\begin{aligned}
& f\left(e, \ldots, e, f(e, \ldots, e), e, \ldots, e, t_{1}, e, \ldots, e\right)=e, \\
& g\left(e, \ldots, e, f(e, \ldots, e), e, \ldots, e, t_{2}, e, \ldots, e\right)=e,
\end{aligned}
$$

with, $f(e, \ldots, e)$ and $g(e, \ldots, e)$ at the $i-t h$ place and $t_{1}, t_{2}$ at the $j-t h$ place.

Furthermore, for $k$ either $i$ or $j$, and any $b \in A$, the following equations

$$
\begin{gathered}
f\left(e, \ldots, e, f\left(e, \ldots, e, x_{1}, e, \ldots, e\right), e, \ldots, e, t_{1}, e, \ldots, e\right)=b, \\
g\left(e, \ldots, e, g\left(e, \ldots, e, x_{2}, e, \ldots, e\right), e, \ldots, e, t_{2}, e, \ldots, e\right)=b
\end{gathered}
$$


with $x_{1}, x_{2}$ at the $k-t$ th place, have unique solutions. Hence, $e$ is a $J$-regular with respect to the pair operation $\left(f^{*}, g^{*}\right)$ on $A$, defined by

$$
\begin{gathered}
f^{*}\left(x_{1}, \ldots, x_{n}\right)=f\left(e, \ldots, e, f\left(x_{1}, \ldots, x_{n}\right), e, \ldots, e, t_{1}, e, \ldots, e\right), \\
g^{*}\left(x_{1}, \ldots, x_{n}\right)=g\left(e, \ldots, e, g\left(x_{1}, \ldots, x_{n}\right), e, \ldots, e, t_{2}, e, \ldots, e\right) .
\end{gathered}
$$

So, by lemma 3.3, the pair operation $\left(f^{*}, g^{*}\right)$ is co-medial with $e$, as an idempotent element. Thus, by corollary 2.5 , there is a commutative semigroup, $(A,+)$, with the unit element $e$ such that

$$
f^{*}\left(x_{1}, \ldots, x_{n}\right)=g^{*}\left(x_{1}, \ldots, x_{n}\right)=\alpha_{1} x_{1}+\cdots+\alpha_{n} x_{n},
$$

where, $\alpha_{1}, \ldots, \alpha_{n}$ are commuting endomorphisms of $(A,+)$.

Again, by the results of the previous section, the mappings

$$
\begin{aligned}
\varphi^{-1}: x \rightarrow f\left(e, \ldots, e, x, e, \ldots, e, t_{1}, e, \ldots, e\right) \\
\psi^{-1}: x \rightarrow g\left(e, \ldots, e, x, e, \ldots, e, t_{2}, e, \ldots, e\right),
\end{aligned}
$$

are bijections on $A$. Thus,

$$
\begin{gathered}
f\left(x_{1}, \ldots, x_{n}\right)=\varphi f^{*}\left(x_{1}, \ldots, x_{n}\right), \\
g\left(x_{1}, \ldots, x_{n}\right)=\psi g^{*}\left(x_{1}, \ldots, x_{n}\right) .
\end{gathered}
$$

Lemma 3.5 Let $(A,+)$ be a commutative semigroup with a unit element and $\varphi_{1}, \ldots, \varphi_{n}$ are bijections on $A$, such that

$$
\varphi_{1}\left(x_{11}+\cdots+x_{1 n}\right)+\cdots+\varphi_{n}\left(x_{n 1}+\cdots+x_{n n}\right)=\varphi_{1}\left(x_{11}+\cdots+x_{n 1}\right)+\cdots+\varphi_{n}\left(x_{1 n}+\cdots+x_{n n}\right) .
$$

Then, there is an automorphism $\eta$ of $(A,+)$ and fixed elements $c_{1}, \ldots, c_{n}$ such that for each $i$, we have:

$$
\varphi_{i} x=\eta x+c_{i},
$$

for all $x \in A$.

Proof: Let $(A,+)$ be a commutative semigroup with a unit element, $e$. In the equation (4), for fixed $i$ and all $j$ except $j=1$, put $x_{i j}=\varphi_{i}^{-1} e$ and all other $x_{p q}$ be unit element except, $x_{1 i}$ and $x_{i 1}$, then we have:

$$
\varphi_{1} x_{1 i}+\varphi_{i} x_{i 1}=\varphi_{1} x_{i 1}+\varphi_{i} x_{1 i}
$$

So, if $x_{1 i}=\varphi_{1}^{-1} e$, then

$$
\varphi_{i} x_{i 1}=\varphi_{1} x_{i 1}+\varphi_{i} \varphi_{1}^{-1} e
$$

for all $x_{i 1} \in A$.

Since, $\varphi_{1}, \varphi_{i}$ are permutations on $(A,+)$, for all $x \in A$ we have:

$$
\varphi_{i} x=\varphi_{1} x+k_{i}
$$

where, $k_{i}$ is a fixed regular element of $(A,+)$. Substituting for the $\varphi_{i}$ in the equation (4) and cancelling the $k_{i}$, which we may do since they are regular elements, we get

$$
\varphi_{1}\left(x_{11}+\cdots+x_{1 n}\right)+\cdots+\varphi_{1}\left(x_{n 1}+\cdots+x_{n n}\right)=\varphi_{1}\left(x_{11}+\cdots+x_{n 1}\right)+\cdots+\varphi_{1}\left(x_{1 n}+\cdots+x_{n n}\right) .
$$

In the equation (5), let $x_{i i}=\varphi_{1}^{-1} e$, where, $i \neq 1,2$, and all other $x_{i j}$ be the unit element, $e$, except, $x_{11}, x_{12}$. Then, we have:

$$
\varphi_{1}\left(x_{11}+x_{12}\right)+\varphi_{1} e=\varphi_{1} x_{11}+\varphi_{1} x_{12}
$$

for all $x_{11}, x_{12}$.

So, if $x_{11}=x_{12}=\varphi_{1}^{-1} e$, then

$$
\varphi_{1}\left(\varphi_{1}^{-1} e+\varphi_{1}^{-1} e\right)+\varphi_{1} e=e,
$$

it means that, $\varphi_{1} e$ has an additive inverse and hence is a regular element. 
Now, we define a bijection $\eta$ on $A$ by the following

$$
\varphi_{1} x=\eta x+\varphi_{1} e,
$$

for all $x \in A$. It follows immediately that, $\eta$ is an automorphism of $(A,+)$.

Hence,

$$
\varphi_{i} x=\varphi_{1} x+k_{i}=\eta x+\varphi_{1} e+k_{i}=\eta x+c_{i},
$$

where, $c_{i}=\varphi_{1} e+k_{i}$, as the sum of two regular elements is a regular element.

Theorem 3.6 Let $(A, f, g)$ be a regular co-medial algebra, then there is a commutative semigroup $(A,+)$ with an unit element, such that

$$
\begin{aligned}
& f\left(x_{1}, \ldots, x_{n}\right)=\gamma_{1} x_{1}+\cdots+\gamma_{n} x_{n}+d_{1}, \\
& g\left(x_{1}, \ldots, x_{n}\right)=\lambda_{1} x_{1}+\cdots+\lambda_{n} x_{n}+d_{2},
\end{aligned}
$$

where, $d_{1}, d_{2}$ are fixed regular elements in $(A,+)$ and $\gamma_{1}, \ldots, \gamma_{n}, \lambda_{1}, \ldots, \lambda_{n}$, are commuting automorphisms of the semigroup $(A,+)$.

Proof: Let $(A, f, g)$ be a regular co-medial algebra, by Lemma 3.4, we know that there is a commutative semigroup with an unit element $e$, such that

$$
\begin{aligned}
& f\left(x_{1}, \ldots, x_{n}\right)=\varphi\left(\alpha_{1} x_{1}+\cdots+\alpha_{n} x_{n}\right), \\
& g\left(x_{1}, \ldots, x_{n}\right)=\psi\left(\alpha_{1} x_{1}+\cdots+\alpha_{n} x_{n}\right),
\end{aligned}
$$

where, $\alpha_{1}, \ldots, \alpha_{n}$, are pairwise commuting endomorphisms of $(A,+)$ and $\varphi, \psi$ are bijections on $A$.

Since, the operation $f$ is co-medial we have:

$$
\begin{array}{r}
\varphi\left(\alpha_{1} \varphi\left(\alpha_{1} x_{11}+\cdots+\alpha_{n} x_{1 n}\right)+\cdots+\alpha_{n} \varphi\left(\alpha_{1} x_{n 1}+\cdots+\alpha_{n} x_{n n}\right)\right)= \\
\varphi\left(\alpha_{1} \varphi\left(\alpha_{1} x_{11}+\cdots+\alpha_{n} x_{n 1}\right)+\cdots+\alpha_{n} \varphi\left(\alpha_{1} x_{1 n}+\cdots+\alpha_{n} x_{n n}\right)\right) .
\end{array}
$$

So,

$$
\begin{array}{r}
\alpha_{1} \varphi \alpha_{1}^{-1}\left(\alpha_{1} \alpha_{1} x_{11}+\cdots+\alpha_{1} \alpha_{n} x_{1 n}\right)+\cdots+\alpha_{n} \varphi \alpha_{n}^{-1}\left(\alpha_{n} \alpha_{1} x_{n 1}+\cdots+\alpha_{n} \alpha_{n} x_{n n}\right)= \\
\alpha_{1} \varphi \alpha_{1}^{-1}\left(\alpha_{1} \alpha_{1} x_{11}+\cdots+\alpha_{1} \alpha_{n} x_{n 1}\right)+\cdots+\alpha_{n} \varphi \alpha_{n}^{-1}\left(\alpha_{n} \alpha_{1} x_{1 n}+\cdots+\alpha_{n} \alpha_{n} x_{n n}\right),
\end{array}
$$

since $\varphi$ is bijection.

Let, $\beta_{i}=\alpha_{i} \varphi \alpha_{i}^{-1}$ and $\alpha_{i} \alpha_{j} x_{i j}=y_{i j}$, then by substitution and since, $\alpha_{1}, \ldots, \alpha_{n}$ are commuting automorphisms of the commutative semigroup $(A,+)$, we have:

$$
\begin{array}{r}
\beta_{1}\left(y_{11}+\cdots+y_{1 n}\right)+\cdots+\beta_{n}\left(y_{n 1}+\cdots+y_{n n}\right)= \\
\beta_{1}\left(y_{11}+\cdots+y_{n 1}\right)+\cdots+\beta_{n}\left(y_{1 n}+\cdots+y_{n n}\right) .
\end{array}
$$

So, by preceding lemma, there is an automorphism $\eta$ of the semigroup $(A,+)$, and regular elements $c_{1}, \ldots, c_{n}$, such that

$$
\begin{aligned}
& \alpha_{i} \varphi \alpha_{i}^{-1} x=\eta x+c_{i}, \\
& \varphi x=\alpha_{i}^{-1} \eta \alpha_{i} x+\alpha_{i}^{-1} \alpha_{i} c_{i}, \\
& \varphi x=\sigma x+d_{1},
\end{aligned}
$$

where, $\sigma=\alpha_{i}^{-1} \eta \alpha_{i}$ is an automorphism of the semigroup and $d_{1}=\alpha_{i}^{-1} \alpha_{i} c_{i}$ is a fixed regular element in $(A,+)$.

Hence,

$$
f\left(x_{1}, \ldots, x_{n}\right)=\gamma_{1} x_{1}+\cdots+\gamma_{n} x_{n}+d_{1},
$$

where, $\gamma_{i}=\sigma \alpha_{i}$ is an automorphism of the semigroup.

Similarly,

$$
g\left(x_{1}, \ldots, x_{n}\right)=\lambda_{1} x_{1}+\cdots+\lambda_{n} x_{n}+d_{2} .
$$

It is easy to check that, $\gamma_{1}, \ldots, \gamma_{n}, \lambda_{1}, \ldots, \lambda_{n}$, are commuting automorphisms of the semigroup $(A,+)$.

The following representation of a medial $n$-ary groupoid was obtained by (Evans, T., 1963). 
Corollary 3.7 Let $(A, f)$ be a medial $n$-ary groupoid with a $i$ - and $j$-regular element, then there exists a commutative semigroup $(A,+)$, such that

$$
f\left(x_{1}, \ldots, x_{n}\right)=\gamma_{1} x_{1}+\cdots+\gamma_{n} x_{n}+d,
$$

where, $d$ is a fixed regular element in $(A,+)$ and $\gamma_{1}, \ldots, \gamma_{n}$ are commuting automorphisms of the semigroup $(A,+)$.

\section{References}

Aczél, J. (1947). The notion of mean values. Det Kogelige Norske Videnskabers Forbandlinger, 19, 83-86.

Bruck, R. H. (1944). Some results in the theory of quasigroups. Transactions of the American Math. Soc., 55, 19-52.

Dudek, W. A., \& Trokhimenko, V. S. (2010). Algebras of multiplace functions. Kremenchug.

Evans, T. (1963). Abstract mean value. Duke Math J., 30, 331-349. http://dx.doi.org/10.1215/S0012-7094-63-03035-7

Hosszu, M. (1953). A generalization of the functional equation of bisymmetry. Studia Mathematica, 14, 100-106.

Ježek, J., \& Kepka, T. (1983). Medial groupoids. Monograph of Acad. Praha, 93/2.

Kolmogorov, A. (1930). Sur la notion de la moyenne. Alti della Royal Academia Nazionale dei Lincei, 12, 388-391.

Kurosh, A. G. (1947). General algebra. M. Nauka. (Russian).

Movsisyan, Yu. M. (1999). Generalization of Toyoda theoram. Proceeding of the Loops'99, Prague.

Movsisyan, Yu. M. (1990). Hyperidentities and Hypervarieties in Algebras. Yerevan: Yerevan State University Press. (Russian).

Movsisyan, Yu. M. (1998). Hyperidentities in algebras and varieties. Uspekhi Math. Nauk, 53, 61-114. English translation in: Russian Math. Surveys, 53, No. 1, 57-108.

Movsisyan, Yu. M. (1986). Introduction to the Theory of Algebras with Hyperidentities. Yerevan: Yerevan State University Press. (Russian).

Movsisyan, Yu. M., \& Nazari, E. (2011). Transitive Modes. Demonstratio Mathematica, XLIV, No. 3, 511-522.

Nagumo, M. (1930). Uber eine Klasse der Mittelwere. Japaness Journal of Mathematics, 7, 71-79.

Romanowska, A., \& Smith, J. D. H. (2002). Modes. World Scientific. 